\title{
Discrete groups with finite decomposition complexity
}

\author{
Erik Guentner, Romain Tessera and Guoliang Yu*
}

\begin{abstract}
In an earlier work we introduced a geometric invariant, called finite decomposition complexity (FDC), to study topological rigidity of manifolds. In particular, we proved the stable Borel conjecture for a closed aspherical manifold whose universal cover, or equivalently whose fundamental group, has FDC. In this note we continue our study of FDC, focusing on permanence and the relation to other coarse geometric properties. In particular, we prove that the class of FDC groups is closed under taking subgroups, extensions, free amalgamated products, HNN extensions, and direct unions. As consequences we obtain further examples of FDC groups - all elementary amenable groups and all countable subgroups of almost connected Lie groups have FDC.
\end{abstract}

Mathematics Subject Classification (2010). 52C45, 20F69.

Keywords. Coarse geometry, decomposition complexity, groups acting on trees, amenable groups, linear groups.

\section{Introduction}

The geometric concept of finite decomposition complexity (FDC) was introduced to study questions concerning the topological rigidity of manifolds [GTY]. Being a coarse geometric property, FDC naturally arises in the following context at the border of large-scale geometry and topology. The bounded Borel conjecture asks the following: Is a quasi-isometry between uniformly contractible Riemannian manifolds necessarily a bounded distance from a homeomorphism? In dimensions higher than four, the powerful tools of surgery theory reduce this problem to proving the bounded Farrell-Jones isomorphism conjecture - a coarse geometric analogue of the usual Farrell-Jones conjecture - which asserts that a certain assembly map in bounded $L$-theory is an isomorphism. We defined FDC for the purpose of developing a large scale cutting and pasting method to attack these conjectures with the help of the controlled Mayer-Vietoris sequence of Ranicki-Yamasaki [RY1], [RY2]. In [GTY] we prove that if the fundamental group of a closed aspherical ${ }^{1}$ manifold has finite

\footnotetext{
*The authors were partially supported by grants from the U.S. National Science Foundation.

${ }^{1}$ Recall that the universal cover of a closed aspherical manifold is uniformly contractible.
} 
decomposition complexity, then its universal cover is boundedly rigid, that is, satisfies the bounded Borel conjecture, and the manifold itself is stably rigid. Our technique extends the controlled cutting and pasting method introduced in [Y1] to compute operator $K$-theory and study the Novikov conjecture under the hypothesis of finite asymptotic dimension.

Here, we shall focus on the FDC property itself, rather than on applications. We shall work primarily in the setting of countable groups, equipped with proper leftinvariant metrics. Recall that every countable group admits such a metric, and that any two such metrics are coarsely equivalent. As finite decomposition complexity is a coarse invariant, the statement that a countable group has finite decomposition complexity is independent of the choice of metric. Our permanence results are summarized in the following theorem.

Theorem. The collection of countable groups having finite decomposition complexity is closed under the formation of subgroups, extensions, free amalgamated products, $\mathrm{HNN}$ extensions and direct unions.

The precise definition of FDC is inspired by the property of finite asymptotic dimension introduced by Gromov [G1] and it is interesting to note that the class of groups having finite asymptotic dimension satisfies the same stability results as above [BD2], [DS] except for direct unions. Indeed, an infinite sum of copies of $\mathbb{Z}$ has FDC whereas it does not have finite asymptotic dimension.

For the next statement, recall that a Lie group is almost connected if it has finitely many connected components.

Theorem. The collection of countable groups having finite decomposition complexity contains all countable subgroups of $\mathrm{GL}(n, R)$, where $R$ is any commutative ring, all countable subgroups of an almost connected Lie group, all hyperbolic groups and all elementary amenable groups.

At the moment, we know of no group not having finite decomposition complexity other than Gromov's examples of (random) groups which do not coarsely embed into a Hilbert space [G2], [G3], [AD]. Since these groups do not coarsely embed into Hilbert space they do not have Property A and hence, according to the following result, do not have FDC:

Theorem. Countable FDC groups have Property A.

On the other hand:

Theorem. Countable groups with finite asymptotic dimension have FDC.

Hence, finite decomposition complexity appears as a generalization of finite asymptotic dimension. Let us emphasize that in general, solvable groups, or linear groups may have infinite asymptotic dimension. 
Organization and remarks. In Section 2 we introduce finite decomposition complexity and outline its basic properties. This section overlaps with Section 2 of [GTY]. In the subsequent section we develop the permanence characteristics of finite decomposition complexity. In Section 4 we show that a metric space having finite asymptotic dimension has finite decomposition complexity. In particular hyperbolic groups have FDC. We then show that finite decomposition complexity implies Property A. As a consequence, a sequence of expanding graphs (viewed as a metric space) does not have finite decomposition complexity.

Section 5 is devoted to examples. We first prove that all (countable) elementary amenable groups have finite decomposition complexity. In the balance of the section we provide complements to [GTY], Theorem 3.0.1, in which we proved that (countable) subgroups of $\mathrm{GL}(n, R)$ have finite decomposition complexity, when $R$ is a domain. Here, we extend this result to the case of an arbitrary commutative ring $R$ with unit. We also provide, for convenience of the reader, a short and self-contained proof in the special case $R=\mathbb{Z}\left[X_{1}, \ldots, X_{m}\right]$ and a proof that $\operatorname{GL}(n, R)$ has finite asymptotic dimension when $R=\mathbb{F}_{q}\left[X_{1}, \ldots, X_{m}\right]$.

\section{Decomposition complexity}

As described in the introduction, finite decomposition complexity arises as a generalization of the notion of finite asymptotic dimension, introduced by Gromov [G1]. Recall that a metric space $X$ has asymptotic dimension at most $d$ if the following condition holds: for every $r>0$ the space $X$ may be written as a union of $d+1$ subspaces, each of which may be further decomposed as an $r$-disjoint union:

$$
X=\bigsqcup_{i=0}^{d} X_{i}, \quad X_{i}=\bigsqcup_{r \text {-disjoint }} X_{i j},
$$

in which the family $\left\{X_{i j}\right\}$ (as both $i$ and $j$ vary) is bounded. ${ }^{2}$ It is frequently useful to think of the integers $0,1, \ldots, d$ as representing colors and as the space $X$ as having been covered by colored sets, any two sets of the same color being at a distance at most $r$. If there exists a $d \in \mathbb{N}$ for which $X$ has asymptotic dimension at most $d$ then $X$ has finite asymptotic dimension.

Consider now the group $G=\bigoplus \mathbb{Z}$ (countably infinite direct sum). We equip $G$ with the left-invariant metric associated to the proper length function

$$
\ell(a)=\sum n\left|a_{n}\right|, \quad \text { where } a=\left(a_{n}\right)_{n=1}^{\infty} .
$$

The group $G$ contains $\mathbb{Z}^{n}$ as a subgroup, for every $n$. Since the asymptotic dimension of $\mathbb{Z}^{n}$ is $n$, it is elementary to see that $G$ does not have finite asymptotic dimension.

\footnotetext{
${ }^{2}$ Here, and subsequently, when $Z$ is a metric space the notation $Z=\sqcup Z_{j}$ ( $r$-disjoint) means that $Z$ is the union of the $Z_{j}$, and that the distance in $Z$ between distinct $Z_{j}$ 's is at least $r$.
} 
Nevertheless, we can still decompose $G$ into bounded pieces in a manner similar to (2.1), but in a two step process. First, we set $d_{1}=0$; given $r_{1} \in \mathbb{N}$ we decompose as

$$
G=G_{0}, \quad G_{0}=\bigsqcup_{r_{1} \text {-disjoint }} x \cdot \mathbb{Z}^{r_{1}}
$$

the disjoint union is as cosets of the subgroup $\mathbb{Z}^{r_{1}} \subset G$ consisting of those elements supported on the first $r_{1}$ coordinates. Second, we set $d_{2}=r_{1}$; given $r_{2} \in \mathbb{N}$ we decompose, using the fact that the asymptotic dimension of $\mathbb{Z}^{r_{1}}$ is (at most) $r_{1}$, as

$$
\mathbb{Z}^{r_{1}}=\bigcup_{i=0}^{d_{2}} X_{i}, \quad X_{i}=\bigsqcup_{r_{2} \text {-disjoint }} X_{i j} .
$$

Observe that since we use a left-invariant metric, decomposing the single space $\mathbb{Z}^{r_{1}}$ gives a decomposition as in (2.1) of every coset $x \cdot \mathbb{Z}^{r_{1}}$ appearing in (2.2) with a uniform bound on the size of the pieces. What is important here is that our success in decomposing $G$ is not affected by the amount of disjointness $r_{1}$ and $r_{2}$ required at each step. Essentially, we have described here a simple case of the metric decomposition game which motivates the definition of (weak) finite decomposition complexity. We shall now proceed to the formal definitions, focusing on finite decomposition complexity - we shall conclude with a brief discussion of weak FDC.

2.1. Definition of FDC. As is clear from the previous discussion, it is advantageous to formulate our definitions not for individual metric spaces but for (countable) families of metric spaces - such families appear naturally in the process of decomposing a space. We shall denote such a metric family by $X=\{X\}$ and shall view a single metric space as a metric family with a single element.

In defining finite decomposition complexity we shall consider decompositions very much like those appearing in the definition of finite asymptotic dimension (2.1); however, for technical reasons related to the applications in [GTY], we shall require $d=1$ in (2.1). ${ }^{3}$ The following basic definitions appear in [GTY] as Definitions 2.0.1, 2.0.2 and 2.0.3.

2.1.1 Definition. An $r$-decomposition of a metric space $X$ over a metric family $y$ is a decomposition

$$
X=X_{0} \cup X_{1}, \quad X_{i}=\bigsqcup_{r \text {-disjoint }} X_{i j},
$$

where each $X_{i j} \in \mathcal{Y}$. A metric family $\mathcal{X}$ is $r$-decomposable over $\mathcal{Y}$ if every member of $\mathcal{X}$ admits an $r$-decomposition over $\mathcal{Y}$. We introduce the notation $X \stackrel{r}{\rightarrow} y$ to indicate that $\mathcal{X}$ is $r$-decomposable over $\mathcal{Y}$.

\footnotetext{
${ }^{3}$ At this point the reader may wish to 'replay' the decomposition game for $\bigoplus \mathbb{Z}$ using only decompositions in which $d=1$.
} 
2.1.2 Definition. Let $\mathfrak{U}$ be a collection of metric families. A metric family $\mathcal{X}$ is decomposable over $\mathfrak{A}$ if, for every $r>0$, there exists a metric family $\mathcal{Y} \in \mathfrak{U}$ and an $r$-decomposition of $\mathcal{X}$ over $\mathcal{Y}$. The collection $\mathfrak{A}$ is stable under decomposition if every metric family which decomposes over $\mathfrak{U}$ actually belongs to $\mathfrak{A}$.

A metric family $\mathcal{Z}$ is bounded if there is a uniform bound on the diameter of its elements:

$$
\sup \{\operatorname{diam}(Z): Z \in Z\}<\infty .
$$

2.1.3 Definition. The collection $\mathfrak{D}$ of metric families with finite decomposition complexity is the minimal collection of metric families containing the bounded metric families and stable under decomposition. We abbreviate membership in $\mathfrak{D}$ by saying that a metric family in $\mathfrak{D}$ has FDC.

Observe that the definitions immediately imply that a metric space with asymptotic dimension at most one has FDC - further, metric families with asymptotic dimension at most one uniformly in the sense of Bell and Dranishnikov [BD1] have FDC. The question of whether metric spaces of finite asymptotic dimension have FDC is more subtle and will be taken up later.

2.2. Equivalent formulations of FDC. We have chosen the most direct route to our definition of FDC. We shall now present two equivalent descriptions of the collection of families having FDC which we require. The first of our equivalent descriptions is based on the following definition, repeated here from [GTY], Section 2.

2.2.1 Definition. We define, for each ordinal $\alpha$, a collection of metric families according to the following prescription:

(1) Let $\mathfrak{D}_{0}$ be the collection of bounded families:

$$
\mathfrak{D}_{0}=\{X: X \text { is bounded }\} .
$$

(2) If $\alpha$ is an ordinal greater than 0 , let $\mathfrak{D}_{\alpha}$ be the collection of metric families decomposable over $\bigcup_{\beta<\alpha} \mathfrak{D}_{\beta}$ :

$$
\mathfrak{D}_{\alpha}=\left\{X \text { : for all } r \text { there exist } \beta<\alpha \text { and } y \in \mathfrak{D}_{\beta} \text { such that } \mathcal{X} \stackrel{r}{\rightarrow} y\right\} .
$$

We introduce the notation $\mathfrak{D}_{\text {fin }}$ for the union of the $\mathfrak{D}_{n}$, over $n \in \mathbb{N}$.

It is instructive to return to the example of $\bigoplus \mathbb{Z}$ in the context of these definitions. As is the case for any metric space having asymptotic dimension at most one, $\mathbb{Z} \in \mathfrak{D}_{1}$. A simple induction reveals that $\mathbb{Z}^{n} \in \mathfrak{D}_{n}$ and looking at the definitions we see that $\bigoplus \mathbb{Z} \in \mathfrak{D}_{\omega}$.

2.2.2 Theorem. A metric family has finite decomposition complexity precisely when it belongs to $\mathfrak{D}_{\alpha}$ for some countable ordinal $\alpha$. 
Our second equivalent description of the collection of families having FDC is based on the idea of a winning strategy for the metric decomposition game. Understanding this description is important for understanding the proofs of the permanence results we shall present below. As a consequence we shall go carefully into the details.

The metric decomposition game has two players, a defender and a challenger. The defender attempts to decompose metric families (as in Definition 2.1.1) in response to requests from the challenger for (presumably large) amounts of disjointness. More formally, let $\mathcal{X}=\mathcal{Y}_{0}$ be the starting family. On the first turn the challenger asserts an integer $r_{1}$, thereby requesting an $r_{1}$-decomposition of $\mathscr{Y}_{0}$; the defender responds by exhibiting an $r_{1}$-decomposition of $y_{0}$ over a new metric family $y_{1}$. On the second turn, the challenger asserts an integer $r_{2}$, thereby requesting an $r_{2}$-decomposition of $y_{1}$; the defender responds by exhibiting an $r_{2}$-decomposition of $\mathcal{y}_{1}$ over a new metric family $y_{2}$. The game continues in this way, turn after turn, and ends if and when the defender produces a bounded family. In this case the defender has won.

A winning strategy is a set of instructions which, if followed by the defender, guarantee victory no matter what requests are made by the challenger. A complete game in which the defender follows the winning strategy produces a series of decompositions:

$$
\mathcal{X}=y_{0} \stackrel{r_{1}}{\longrightarrow} y_{1} \stackrel{r_{2}}{\longrightarrow} y_{2} \longrightarrow \cdots \stackrel{r_{n}}{\longrightarrow} y_{n}, \quad y_{n} \text { bounded }
$$

When the defender follows a winning strategy the eventual outcome of the game is certain. Nevertheless, the number of turns required may not be known and may depend on the requests made by the challenger. Indeed, the required number of turns may be unbounded, as is already the case for the winning strategy inherent in the discussion of our example $\bigoplus Z$.

A winning strategy may or may not exist; when it does, we say that the original metric family $\mathcal{X}$ admits a decomposition strategy. We have arrived at our second description of FDC. ${ }^{4}$

2.2.3 Theorem. A metric family has finite decomposition complexity precisely when it admits a decomposition strategy.

We shall work directly with decomposition strategies in the proof of this theorem, as well as in the proofs of some of our permanence properties, and so provide their precise mathematical formulation. The idea is to encode the turns of possible decomposition games as a labeled tree - each turn of a possible game corresponds to a vertex; the metric family relevant to a particular turn labels the corresponding vertex; from each vertex emanate edges labeled with the possible requests of the challenger and the defender responds by following the appropriate edge.

Formally, a decomposition tree is a directed, rooted tree $T$ satisfying the following:

(1) every non-root vertex of $T$ is the terminal vertex of a unique edge;

\footnotetext{
${ }^{4}$ Taken together, Theorems 2.2.2 and 2.2.3 form Theorem 2.2.1 of [GTY].
} 
(2) every non-leaf vertex of $T$ is the initial vertex of countably many edges, which are labeled by the natural numbers;

(3) $T$ contains no infinite ray (geodesic edge-path).

A decomposition strategy for a metric family $\mathcal{X}$ comprises a decomposition tree $T$, the support tree of the strategy, together with a labeling of the vertices of $T$ by metric families $y$ subject to the following requirements:

(4) the root vertex of $T$ is labeled $X$;

(5) every leaf of $T$ is labeled by a bounded family;

(6) if $y$ labels the initial vertex and $Z$ the terminal vertex of an edge labeled by $r \in \mathbb{N}$ then $\mathcal{Y}$ is $r$-decomposable over $\mathcal{Z}$.

Games in which the defender follows the winning strategy correspond to paths in $T$ beginning at its root and ending at a leaf. For example, if the edges along the path are labeled $r_{1}, \ldots, r_{n}$ and the vertices are labeled $\mathcal{X}, Y_{1}, \ldots, y_{n}$ we obtain the series of decompositions in (2.3).

Again, we illustrate these ideas in the context of our example $\bigoplus \mathbb{Z}$. It will be convenient to denote the interval $\{0,1, \ldots, r\} \subset \mathbb{Z}$ by $[r]$. Further, we shall denote a metric family in which all spaces are isometric to a single space by that space.

Tiling $\mathbb{Z}$ with translates of the interval $[r]$, and coloring the translates alternately red and blue gives an $r$-decomposition of $\mathbb{Z}$ over a bounded family. The diagram on the left in Figure 1 expresses this as a decomposition strategy. A strategy for $\mathbb{Z}^{2}$ is obtained by first applying the strategy for $\mathbb{Z}$ in one factor, and then in the other. The decomposition game ends after two turns, with $\mathbb{Z}^{2}$ being decomposed into translates of the product $\left[r_{2}\right] \times\left[r_{1}\right]$ of intervals. This strategy is depicted in the middle diagram in Figure 1. Continuing in this way, we obtain a strategy for $\mathbb{Z}^{n}$ in which the defender

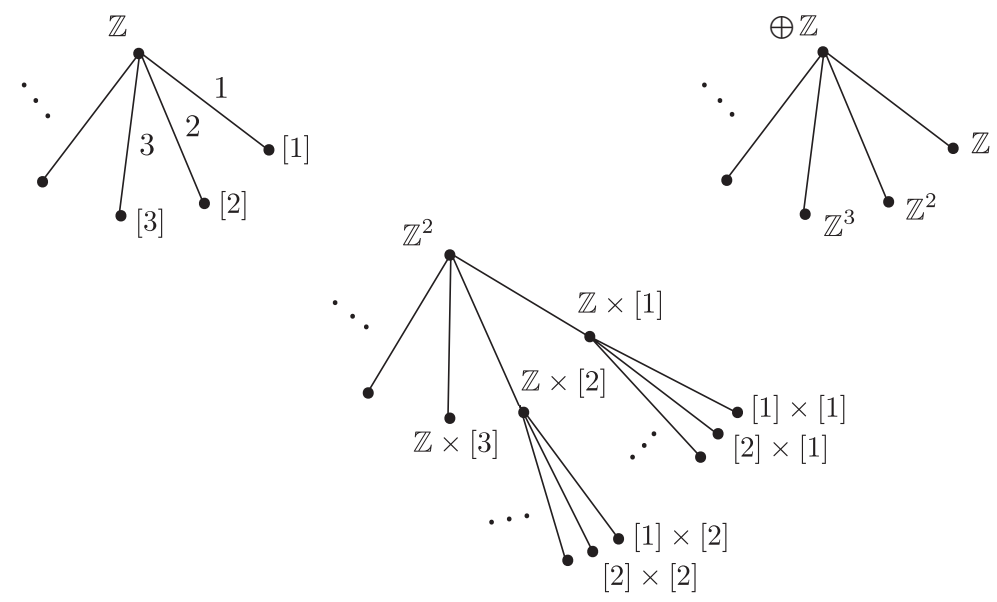

Figure 1. Strategies for $\mathbb{Z}, \mathbb{Z}^{2}$ and $\bigoplus \mathbb{Z}$. 
wins in $n$ turns, no matter how the challenger plays. Paths in this strategy beginning with $\mathbb{Z}^{n}$ and ending in a bounded family have the form

$$
\mathbb{Z}^{n} \stackrel{r_{1}}{\longrightarrow} \mathbb{Z}^{n-1} \times\left[r_{1}\right] \longrightarrow \cdots \stackrel{r_{n}}{\longrightarrow}\left[r_{n}\right] \times \cdots \times\left[r_{1}\right]
$$

Finally, we build a strategy for $\bigoplus \mathbb{Z}$ as follows. Begin with the diagram on the right in Figure 1, which represents the decomposition of $\bigoplus \mathbb{Z}$ into cosets for the various $\mathbb{Z}^{r}$ described earlier. Then, to each leaf attach the strategy for the appropriate $\mathbb{Z}^{r}$. Observe that in this strategy the number of turns required for the defender to win is unbounded, and depends on the first request of the challenger.

We turn to the proofs of Theorems 2.2.2 and 2.2.3. We require the following lemma.

2.2.4 Lemma. Let $T$ be a decomposition tree. There exists a function $v \mapsto \alpha_{v}$ from the set of vertices of $T$ to a set of countable ordinal numbers with the properties that $\alpha_{v}=0$ if $v$ is a leaf and

$$
\alpha_{v}=\sup _{w<v}\left\{\alpha_{w}+1\right\}
$$

otherwise.

Proof. Observe that, by virtue of the no-infinite-ray assumption, a decomposition tree has leaves. Define, for each countable ordinal $\alpha$, a subset $L_{\alpha}$ of the vertex set of $T$ by transfinite recursion: $L_{0}$ is the set of leaves of $T$; for $\alpha>0$,

$$
L_{\alpha}=\text { the set of leaves of } T \backslash \bigcup_{\beta<\alpha} L_{\beta} \text {, }
$$

if this set is non-empty, and $L_{\alpha}=\emptyset$ otherwise. Note that if it is non-empty, the set $T \backslash \bigcup_{\beta<\alpha} L_{\beta}$ is again a decomposition tree, and therefore has leaves.

Let $\alpha_{0}=\left\{\alpha: L_{\alpha} \neq \emptyset\right\}$ and let $\mathfrak{L}=\left\{L_{\alpha}, \alpha<\alpha_{0}\right\}$. Clearly, $\mathfrak{Z}$ is a partition of the set of vertices of $T$, and the map $\alpha \mapsto L_{\alpha}: \alpha_{0} \rightarrow \mathfrak{L}$ is a bijection. It follows that $\alpha_{0}$ is countable. Finally, for every vertex $v$, let $\alpha_{v}$ be the unique $\alpha$ such that $v \in L_{\alpha}$. It is not difficult to see that $\alpha_{v}$ satisfies the desired properties.

Proof of Theorems 2.2.2 and 2.2.3. For purposes of the proof let $\mathfrak{D}^{\prime}$ be collection of families admitting a decomposition strategy; let $\mathfrak{D}^{\prime \prime}$ be the collection of families belonging to $\mathfrak{D}_{\alpha}$ for some countable ordinal $\alpha$. We must show $\mathfrak{D}^{\prime \prime}=\mathfrak{D}^{\prime}=\mathfrak{D}$.

A simple transfinite induction shows that $\mathfrak{D}_{\alpha} \subset \mathfrak{D}$ for every ordinal $\alpha$. Thus, $\mathfrak{D}^{\prime \prime} \subset \mathfrak{D}$.

Next, we show that $\mathfrak{D} \subset \mathfrak{D}^{\prime}$. Since a bounded family trivially admits a decomposition strategy, it suffices to show that the collection $\mathfrak{D}^{\prime}$ is closed under decomposability. Let $\mathcal{X}$ be a family decomposable over $\mathfrak{D}^{\prime}$. For every $r \in \mathbb{N}$, obtain a family $\mathcal{Y}_{r} \in \mathfrak{D}^{\prime}$ such that $\mathcal{X}$ is $r$-decomposable over $\mathcal{Y}_{r}$. A decomposition strategy for $\mathcal{X}$ is 
obtained by attaching strategies for the $y_{r}$ to the bottom of an 'infinite caret' whose root vertex is labeled $\mathcal{X}$ and whose edges are labeled by $\mathbb{N}$ as shown in Figure 2 .

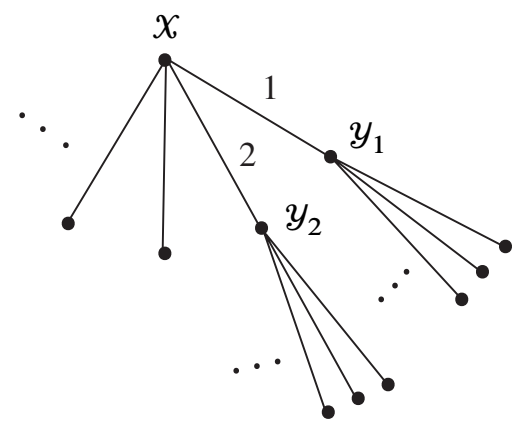

Figure 2. Concatenating strategies.

Finally, we show that $\mathfrak{D}^{\prime} \subset \mathfrak{D}^{\prime \prime}$. Let $\mathcal{X} \in \mathfrak{D}^{\prime}$. Let $T$ be the support tree of a decomposition strategy for $\mathcal{X}$; denote the label of a vertex $v$ by $\mathcal{Y}_{v}$ and let $v \mapsto \alpha_{v}$ be a function with the properties outlined in Lemma 2.2.4. It suffices to show that for every ordinal $\alpha$ we have: if $\alpha_{v} \leq \alpha$ then $y_{v} \in \mathfrak{D}_{\alpha}$. This follows easily by transfinite induction.

2.2.5 Remark. At the outset of this project, we defined a property weaker than FDC which is more transparently related to finite asymptotic dimension, introduced by Gromov [G1]. The difference between this property - weak finite decomposition complexity - and the one defined here lies in the type of decomposition - we replace $r$-decomposability by the notion of $(d, r)$-decomposability.

A metric family $\mathcal{X}$ is $(d, r)$-decomposable over a metric family $\mathcal{Y}$ if every $X \in \mathcal{X}$ admits a decomposition

$$
X=X_{0} \cup \cdots \cup X_{d}, \quad X_{i}=\bigsqcup_{r \text {-disjoint }} X_{i j},
$$

where each $X_{i j} \in \mathcal{Y}$. The metric family $\mathcal{X}$ weakly decomposes over the collection $\mathfrak{A}$ of metric families, if there exists a $d \in \mathbb{N}$ such that for every $r>0$, there exists $y \in \mathfrak{A}$ and a $(d, r)$-decomposition of $\mathcal{X}$ over $\mathcal{Y}$. The collection of metric families with weak finite decomposition complexity is the smallest collection containing bounded metric families, and stable under weak decomposition.

Clearly, both FDC and finite asymptotic dimension (uniformly in the sense of Bell and Dranishnikov [BD1]) imply weak FDC. While true that finite asymptotic dimension implies FDC itself, this is already difficult. (See Theorem 4.1 below.)

2.2.6 Question. Are finite and weak finite decomposition complexity equivalent? 


\section{Permanence of FDC}

In this section we shall study the permanence characteristics of finite decomposition complexity. While we shall focus on finite decomposition complexity, all permanence results hold for weak finite decomposition complexity as well.

We begin by recalling some elementary concepts from coarse geometry. Let $\mathcal{X}$ and $\mathcal{Y}$ be metric families. A subspace of the family $\mathcal{Y}$ is a family $\mathcal{Z}$, every element of which is a subspace of some element of $y$. A map of families from $X$ to $y$ is a collection of functions $F=\{f\}$, each mapping some $X \in X$ to some $Y \in \mathcal{Y}$ and such that every $X \in \mathcal{X}$ is the domain of at least one $f \in F$. We use the notation $F: X \rightarrow Y$ and, when confusion could occur, write $f: X_{f} \rightarrow Y_{f}$ to refer to an individual function in $F$. The inverse image of the subspace $\mathcal{Z}$ is the collection

$$
F^{-1}(\mathcal{Z})=\left\{f^{-1}(Z): Z \in \mathcal{Z}, f \in F\right\} .
$$

The inverse image is a subspace of $\mathcal{X}$.

A map of families $F: \mathcal{X} \rightarrow \mathcal{Y}$ is uniformly expansive if there exists a nondecreasing function $\rho:[0, \infty) \rightarrow[0, \infty)$ such that for every $f \in F$ and every $x, y \in X_{f}$

$$
d(f(x), f(y)) \leq \rho(d(x, y)) ;
$$

it is effectively proper if there exists a proper non-decreasing function $\delta:[0, \infty) \rightarrow$ $[0, \infty)$ such that for every $f \in F$ and every $x, y \in X_{f}$

$$
\delta(d(x, y)) \leq d(f(x), f(y)) ;
$$

it is a coarse embedding if it is both uniformly expansive and effectively proper. (In this case, if $\mathcal{X}$ is unbounded then $\rho$ is also proper.) Summarizing, a map of families $F$ is a coarse embedding if the individual $f$ are coarse embeddings admitting a common $\delta$ and $\rho$. Similar remarks apply to uniformly expansive and effectively proper maps.

Recall that a coarse embedding $f: X \rightarrow Y$ of metric spaces is a coarse equivalence if it admits an 'inverse' - a coarse embedding $g: Y \rightarrow X$ for which the compositions $f \circ g$ and $g \circ f$ are close to the identity maps on $X$ and $Y$, respectively:

$$
\text { there exists } C>0 \text { such that } d(x, g f(x)) \leq C \text { and } d(y, g f(y)) \leq C,
$$

for all $x \in X$ and $y \in Y$. So motivated, a coarse embedding $F: \mathcal{X} \rightarrow \mathcal{Y}$ of metric families is a coarse equivalence if each $f \in F$ is a coarse equivalence admitting an inverse $g$ satisfying the following two conditions: first, the collection $G=\{g\}$ is a coarse embedding $y \rightarrow X$ of metric families; second, the composites $f \circ g$ and $g \circ f$ are uniformly close to the identity maps on the spaces comprising $\mathcal{X}$ and $\mathcal{Y}$, in the sense that the constant $C$ in (3.3) may be chosen independently of the spaces $X \in \mathcal{X}$ and $Y \in \mathcal{Y}$. Two metric families $\mathcal{X}$ and $\mathcal{Y}$ are coarsely equivalent if there exists a coarse equivalence $X \rightarrow Y$. Coarse equivalence is an equivalence relation. 
3.1. Permanence for spaces. The primitive permanence properties for metric families are Coarse Invariance, the Fibering and Union Theorems. We shall prove these in this section.

3.1.1 Lemma. Let $\mathcal{X}$ and $y$ be metric families and let $F: X \rightarrow Y$ be a uniformly expansive map. For every $r>0$ there exists an $s>0$ such that if $\mathcal{Z}$ and $\mathcal{Z}^{\prime}$ are subspaces of $\mathcal{Y}$ and $Z^{\prime} \stackrel{s}{\longrightarrow} \mathcal{Z}$ then $F^{-1}\left(Z^{\prime}\right) \stackrel{r}{\rightarrow} F^{-1}(\mathcal{Z})$. Further, $s$ depends only on $r$ and on the non-decreasing function $\rho$ satisfying (3.1).

Proof. Assuming $F$ is uniformly expansive let $\rho$ be such that (3.1) holds. Set $s=\rho(r)$ and assume $Z^{\prime} \stackrel{s}{\longrightarrow} Z$. An element of $F^{-1}\left(Z^{\prime}\right)$ has the form $f^{-1}(Z)$ for some $Z \in Z^{\prime}$ and $f \in F$. Given such an element obtain a decomposition

$$
Z=Z_{0} \cup Z_{1}, \quad Z_{i}=\bigsqcup_{s \text {-disjoint }} Z_{i j},
$$

in which the $Z_{i j} \in \mathcal{Z}$. We then have a decomposition

$$
f^{-1}(Z)=f^{-1}\left(Z_{0}\right) \cup f^{-1}\left(Z_{1}\right), \quad f^{-1}\left(Z_{i}\right)=\bigcup f^{-1}\left(Z_{i j}\right),
$$

in which the $f^{-1}\left(Z_{i j}\right) \in F^{-1}(\mathcal{Z})$. From the definition of $s$ we see immediately that the union on the right is $r$-disjoint.

3.1.2 Lemma. Let $\mathcal{X}$ and $Y$ be metric families and let $F: X \rightarrow Y$ be an effectively proper map. If $\mathcal{Z}$ is a bounded subspace of $\mathcal{Y}$ then $F^{-1}(\mathcal{Z})$ is a bounded subspace of $\mathcal{X}$.

Proof. Assuming $F$ is effectively proper let $\delta$ be such that (3.2) holds. Let $B$ bound the diameter of the metric spaces in the family $\mathcal{Z}$. Using the hypothesis that $\delta$ is proper, let $A$ be such that $\delta(A) \geq B$. Then $F^{-1}(\mathcal{Z})$ is bounded by $A$.

3.1.3 Coarse Invariance. Let $\mathcal{X}$ and $\mathcal{Y}$ be metric families. If there is a coarse embedding from $\mathcal{X}$ to $\mathcal{Y}$ and $\mathcal{Y}$ has finite decomposition complexity, then so does $\mathcal{X}$. In particular:

(1) a subspace of a metric family with FDC itself has FDC;

(2) if $\mathcal{X}$ and $\mathcal{Y}$ are coarsely equivalent, then $\mathcal{X}$ has FDC if and only if $\mathcal{Y}$ does.

Proof. By pruning and relabeling we can pull back a decomposition strategy for $y$ to $X$. Precisely, select an increasing sequence of natural numbers $s_{1}, s_{2}, \ldots$ such that $s_{i} \geq i$. Prune $T$ by removing a vertex $v$, together with the entire 'downward' subtree based at $v$ and the unique upward edge incident at $v$, when this upward edge is labeled by an element of $\mathbb{N} \backslash\left\{s_{i}\right\}$. The resulting graph $T^{\prime}$ is a subtree of $T$ and a vertex of $T^{\prime}$ is a leaf of $T^{\prime}$ exactly when it is a leaf of $T$. Relabel a typical edge as shown in Figure 3. It follows from Lemmas 3.1.1 and 3.1.2 that the labeling requirements for a decomposition strategy are fulfilled. 

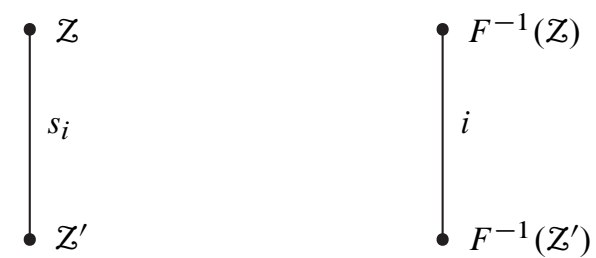

Figure 3. Relabeling.

3.1.4 Fibering Theorem. Let $\mathcal{X}$ and $\mathcal{Y}$ be metric families and let $F: \mathcal{X} \rightarrow \mathcal{Y}$ be a uniformly expansive map. Assume $\mathcal{Y}$ has finite decomposition complexity, and that for every bounded subspace $\mathcal{Z}$ of $\mathcal{Y}$ the inverse image $F^{-1}(\mathcal{Z})$ has finite decomposition complexity. Then $\mathcal{X}$ has finite decomposition complexity.

Proof. A decomposition strategy for $y$ pulls back, as in the previous proof, to a partial decomposition strategy for $\mathcal{X}$. It is partial in that the leaves of its support tree are labeled by families which are not (necessarily) bounded but rather are the inverse images of bounded subspaces of $\mathcal{Y}$. We complete the partial strategy by attaching to a leaf labeled by $F^{-1}(\mathcal{Z})$ a strategy for this family.

3.1.5 Remark. Directly from the definitions we see that $\mathcal{X} \in \mathfrak{D}_{n}$ precisely when $\mathcal{X}$ admits a decomposition strategy in which the strategy tree has depth not greater than $n$, meaning that the length of a geodesic emanating from the root vertex is at most $n$. In the notation of the Fibering Theorem, the previous proof shows the following: suppose that $\mathcal{Y} \in \mathfrak{D}_{n}$ and that there exists a natural number $m$ such that $F^{-1}(\mathbb{Z}) \in \mathfrak{D}_{m}$ for every bounded subspace $\mathcal{Z}$ of $\mathcal{Y}$; then $\mathcal{X} \in \mathfrak{D}_{n+m}$.

3.1.6 Remark. Continuing in the spirit of the previous remark, suppose that $\mathcal{Y} \in \mathfrak{D}_{\text {fin }}$ and that $F^{-1}(\mathcal{Z}) \in \mathfrak{D}_{\text {fin }}$ for every bounded subspace $\mathcal{Z}$ of $\mathcal{Y}$. Then $\mathcal{X} \in \mathfrak{D}_{\omega+\text { fin }}$, meaning that for some natural number $n$ we have $\mathcal{X} \in \mathfrak{D}_{\omega+n}$. The distinction between this remark and the previous is that here we assume merely that each $F^{-1}(\mathbb{Z}) \in \mathfrak{D}_{m}$ for some natural number $m$, which may depend on $\mathfrak{Z}$.

3.1.7 Finite Union Theorem. Let $X$ be a metric space, expressed as a union of finitely many metric subspaces $X=\bigcup_{i=0}^{n} X_{i}$. If the metric family $\left\{X_{i}\right\}$ has finite decomposition complexity so does $X$.

Proof. Consider first the case $n=2$, illustrated in Figure 4. For every $r>0$, the metric space $X=X_{1} \cup X_{2}$ is $r$-decomposable over the family $\left\{X_{1}, X_{2}\right\} \in \mathfrak{D}$. Thus $X \in \mathfrak{D}$. The general case follows by induction.

3.1.8 Union Theorem. Let $X$ be a metric space, expressed as a union of metric subspaces $X=\bigcup_{i \in I} X_{i}$. Suppose that the metric family $\left\{X_{i}\right\}$ has finite decomposition 
complexity and that for every $r>0$ there exists a metric subspace $Y(r) \subset X$ having finite decomposition complexity and such that the subspaces $Z_{i}(r)=X_{i} \backslash Y(r)$ are pairwise $r$-disjoint. Then $X$ has finite decomposition complexity.

Proof. To conclude that $X$ has finite decomposition complexity, it suffices to show that $X$ is decomposable over $\mathfrak{D}$. The proof of this is illustrated in Figure 5. Formally, for every $r>0$ let $Y(r)$ and $Z_{i}(r)$ be as in the statement. The decomposition

$$
X=Y(r) \cup Z(r), \quad Z(r)=\bigsqcup_{r \text {-disjoint }} Z_{i}(r)
$$

is an $r$-decomposition of $X$ over the family $y_{r}=\{Y(r)\} \cup\left\{Z_{i}(r): i \in I\right\}$. Since the $Z_{i}(r)$ are subspaces of the $X_{i}$ and the family $\left\{X_{i}\right\}$ has finite decomposition complexity, the family $\left\{Z_{i}(r): i \in I\right\}$ does as well; since $Y(r)$ has finite decomposition complexity, the family $y_{r}$ does as well.

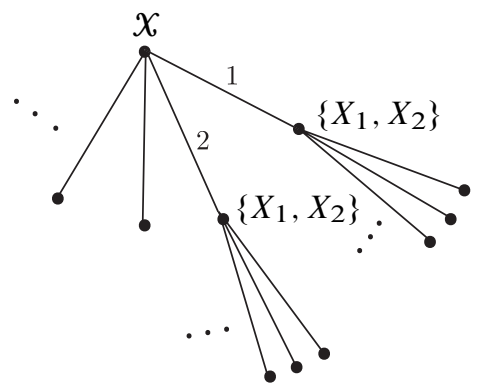

Figure 4. A finite union.

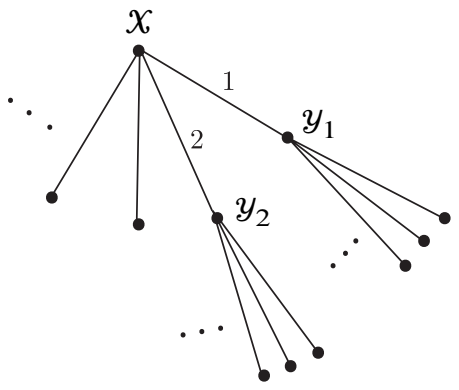

Figure 5. A union.

3.1.9 Remark. While we could state union theorems in the context of metric families (instead of single metric spaces) we shall not require this level of generality.

3.2. Permanence for groups. Most (though not all) permanence properties for discrete groups are deduced by allowing the group to act on an appropriate metric space, and applying the permanence results for spaces detailed in the previous section.

Let $G$ be a countable discrete group. Recall that a countable discrete group admits a proper length function $\ell$ and that any two metrics defined from proper length functions by the formula

$$
d(s, t)=\ell\left(s^{-1} t\right)
$$

are coarsely equivalent (in fact, the identity map is a coarse equivalence). As a consequence, a coarsely invariant property of metric spaces is a property of countable discrete groups - whether or not a group has the property is not an artifact of 
the particular metric chosen. Consequently, we say that a discrete group has finite decomposition complexity if its underlying metric space has finite decomposition complexity for some (equivalently every) metric defined as above.

3.2.1 Proposition. A countable direct union of groups with finite decomposition complexity has finite decomposition complexity. Equivalently, a countable discrete group has finite decomposition complexity if and only if every finitely generated subgroup does.

Proof. Let $G$ be a countable discrete group, expressed as the union of a collection of subgroups each of which has finite decomposition complexity: $G=\cup G_{i}$. Equip $G$ with a proper length function and associated metric. We shall show that for every $r>0$ (the metric space) $G$ is $r$-decomposable over a metric family with finite decomposition complexity.

Let $r>0$. Since the ball of radius $r$ centered at the identity in $G$ is finite there exists $i=i(r)$ such that this ball is contained in $G_{i}$. It follows that the decomposition of $G$ into the cosets of $G_{i}$ is $r$-disjoint. Further, the family comprised of these cosets has finite decomposition complexity since each coset is isometric to $G_{i}$, which has finite decomposition complexity (in any proper metric so in the subspace metric) by assumption.

Let now $X$ be a metric space, and suppose that $G$ acts (by isometries) on $X$. For $R>0$ the $R$-coarse stabilizer of $x$ is

$$
\operatorname{Stab}(x, R)=\{g \in G: d(x, g \cdot x)<R\} .
$$

In general an $R$-coarse stabilizer is a subset of $G$. The 0 -coarse stabilizer of $x$ is its stabilizer, a subgroup of $G$. The space $X$ is locally finite if every ball is finite.

3.2.2 Lemma. For every $x \in X$ the orbit map $g \mapsto g \cdot x: G \rightarrow X$ is uniformly expansive.

3.2.3 Proposition. Let $G$ be a countable discrete group acting on a metric space $X$ with finite decomposition complexity. If there exists $x_{0} \in X$ such that for every $R>0$ the $R$-coarse stabilizer of $x_{0}$ has finite decomposition complexity then $G$ has finite decomposition complexity.

Proof. By restricting to the orbit of $x_{0}$ we may assume the action is transitive. Together with the coarse stabilizer condition, the fact that the orbit map $g \rightarrow g \cdot x_{0}$ is a surjective and equivariant map $G \rightarrow X$ implies that the hypothesis of the Fibering Theorem 3.1.4 are fulfilled. The proposition follows.

3.2.4 Corollary. Let $G$ and $X$ be as in the previous proposition. If $X$ is locally finite, and if there exists $x_{0} \in X$ such that the stabilizer of $x_{0}$ has finite decomposition complexity, then $G$ has finite decomposition complexity. 
Proof. Under the stated hypotheses the Finite Union Theorem implies that the coarse stabilizers of $x_{0}$ have finite decomposition complexity. Thus, the previous proposition applies.

3.2.5 Corollary. The collection of countable discrete groups with finite decomposition complexity is closed under extensions.

3.2.6 Proposition. If a countable discrete group acts (without inversion) on a tree, and the vertex stabilizers of the action have finite decomposition complexity, then the group itself has finite decomposition complexity.

Proof. According to the Bass-Serre theory, a group as in the statement is built from vertex stabilizers of the action by iterated free products (with amalgam), HNN extensions and direct unions. An HNN extension, in turn, is built from free products (with amalgam), a direct union and a group extension. As we have seen that the class of (countable discrete) groups with finite decomposition complexity is closed under direct unions, subgroups and extensions the proposition follows once we show that a free product with amalgam has finite decomposition complexity if the factors do. But, this follows axiomatically from the above proven permanence results - essentially, apply fibering to the action on the Bass-Serre tree using the union theorem to conclude that the coarse stabilizers have finite decomposition complexity. For a more detailed discussion see $[\mathrm{G}]$ and the references therein.

\section{FDC, Property A and finite asymptotic dimension}

In this section we shall discuss how the property of finite decomposition complexity relates to other familiar properties from coarse geometry, notably to Property A and to finite asymptotic dimension.

We have seen that the definition of finite decomposition complexity is motivated by finite asymptotic dimension. We begin by pursuing this discussion further, our goal being to prove that a metric space having finite asymptotic dimension has finite decomposition complexity as well.

Recall that a metric space is proper if closed and bounded sets are compact. A discrete metric space is proper precisely when it is locally finite in the sense that every ball is finite. It is not difficult to see that a proper metric space having finite asymptotic dimension has finite decomposition complexity. Indeed, according to a theorem of Dranishnikov-Zarichnyi a proper metric space having finite asymptotic dimension admits a coarse embedding into the product of finitely many locally finite trees [DZ]. As trees have finite decomposition complexity, we may apply our permanence results to conclude. More generally, for metric spaces which are not necessarily proper we have the following theorem. 
4.1 Theorem. A metric space has finite asymptotic dimension if and only if it belongs to $\mathfrak{D}_{\text {fin. }}$. In particular, a metric space having finite asymptotic dimension has finite decomposition complexity as well.

We are primarily interested in the forward implication, and shall reduce the general case to the case of proper metric spaces using an ultralimit construction. Before turning to the proof, we recall the relevant background notions. Let $X$ be a (pseudo-) metric space. The Gromov triple product (with respect to a base point $x_{0}$ ) is

$$
(x \mid y)=\frac{1}{2}\left(d\left(x, x_{0}\right)+d\left(y, x_{0}\right)-d(x, y)\right) .
$$

The (pseudo)-metric space $X$ is Gromov 0-hyperbolic if

$$
(x \mid z) \geq \min \{(x \mid y),(y \mid z)\},
$$

for all $x, y$ and $z \in X$. The notion of 0 -hyperbolicity is independent of the choice of base point [A], Proposition 2.2. A Gromov 0-hyperbolic (pseudo)-metric space has asymptotic dimension at most 1. (See [R1] for a direct argument.) Hence, a Gromov 0 -hyperbolic (pseudo-)metric space has finite decomposition complexity.

Proof of Theorem 4.1. A simple induction shows that a (pseudo)-metric space belonging to $\mathfrak{D}_{n}$ has asymptotic dimension is at most $2^{n}-1$.

For the converse, let $X$ be a (pseudo)-metric space having finite asymptotic dimension at most $n$. We shall show that $X$ has finite decomposition complexity, indeed that $X \in \mathfrak{D}_{\text {fin }}$. Apply the result of Drashnikov-Zarichnyi [DZ] to the finite subsets of $X$ - these are locally finite metric spaces and the essential observation here is that the result of Drashnikov-Zarichnyi applies uniformly. Precisely, there exists $\rho$ and $\delta$ and for each finite subset $F \subset X$ a $\rho$-uniformly expansive and $\delta$-effectively proper map into a product of trees:

$$
F \rightarrow T_{0}^{F} \times \cdots \times T_{n}^{F} .
$$

Projecting to the individual factors we lift the tree metrics back to $F$ to obtain a family of (pseudo-)metrics $d_{0}^{F}, \ldots, d_{n}^{F}$ on $F$ with the following two properties. First, each $d_{i}^{F}$ is Gromov 0-hyperbolic - recall here that an $\mathbb{R}$-tree is Gromov 0-hyperbolic. Second, the identity $F \rightarrow F$ is $\rho$-uniformly expansive and $\delta$-effectively proper, when the domain is equipped with the subspace metric from $X$ and the range the sum metric $d_{0}^{F}+\cdots+d_{n}^{F}$ - explicitly, for all $x, y \in F$ we have

$$
\delta\left(d_{X}(x, y)\right) \leq d_{0}^{F}(x, y)+\cdots+d_{n}^{F}(x, y) \leq \rho\left(d_{X}(x, y)\right) .
$$

Let now $\mathfrak{F}$ be the collection of finite subsets of $X$ containing a fixed base point $x_{0}$, viewed as a directed set under inclusion. Let $\omega$ be an ultrafilter on the set $\mathfrak{F}$ with the following property: for every convergent net $\left(t_{F}\right)_{F \in \mathfrak{F}}$ of real numbers we have

$$
\lim t_{F}=\omega-\lim t_{F},
$$


where the limit of the left is the ordinary limit of the convergent net, and the limit of the right is the limit with respect to the ultrafilter $\omega$.

For each fixed $i=0, \ldots, n$ form the ultraproduct $X_{i}=\omega-\lim F_{i}$, where we write $F_{i}$ for $F$ equipped with the metric $d_{i}^{F}$. Precisely, $X_{i}$ is the space of of $\mathfrak{F}$-indexed nets $\bar{x}=\left(x_{F}\right)$, with $x_{F} \in F$, for which $d_{i}^{F}\left(x_{F}, x_{0}\right)$ is bounded independent of $F .^{5}$ Define a pseudo-metric on $X_{i}$ by

$$
d_{i}(\bar{x}, \bar{y})=\omega-\lim d_{i}^{F}\left(x_{F}, y_{F}\right),
$$

where $\bar{x}=\left(x_{F}\right)$ and $\bar{y}=\left(y_{F}\right)$ are elements of $X_{i}$. Define a map $\alpha_{i}: X \rightarrow X_{i}$ by associating to $x$ the 'constant sequence'; it follows immediately from (4.1) that

$$
\alpha_{i}(x)_{F}= \begin{cases}x, & x \in F \\ x_{0}, & \text { else }\end{cases}
$$

satisfies the boundedness condition required of elements of $X_{i}$.

Now, the individual $X_{i}$ are Gromov 0-hyperbolic, essentially because the condition for 0-hyperbolicity, satisfied by the individual $d_{i}^{F}$, involves only finitely many points and passes to the limit intact. Thus, each $X_{i}$ has finite decomposition complexity and indeed belongs to $\mathscr{D}_{1}$. An elementary application of permanence shows that the product $X_{0} \times \cdots \times X_{n}$ belongs to $\mathfrak{D}_{n+1}$. See Remark 3.1.5.

The proof concludes with the observation that the product of the $\alpha_{i}$ is a coarse embedding $X \rightarrow X_{0} \times \cdots \times X_{n}$. To verify this observe that for $x \in X$ we have $\alpha_{i}(x)=x$ for $\omega$-almost every $F$. So, if $y \in X$ as well we have

$$
\sum_{i=0}^{n} d_{i}\left(\alpha_{i}(x), \alpha_{i}(y)\right)=\omega-\lim \sum_{i=0}^{n} d_{i}^{F}(x, y)
$$

which by (4.1) is bounded from above by $\rho\left(d_{X}(x, y)\right)$ and from below by $\delta\left(d_{X}(x, y)\right)$.

4.0.7 Remark. We are unable to find a reference for the existence of an ultrafilter as required in the previous proof; we provide instead the following simple argument. In the notation of the proof, the collection of all subsets of $\mathfrak{F}$ containing a set of the form

$$
\left\{F \in \mathfrak{F}: F_{0} \subset F\right\}
$$

is a filter, the filter of tails in $\mathfrak{F}$. An ultrafilter containing the filter of tails is as required - the existence of an ultrafilter containing a given filter is a classic application of Zorn's lemma.

\footnotetext{
${ }^{5}$ As we work with pseudo-metric spaces it is not necessary to consider equivalence classes as would be typical.
} 
We turn now to a discussion of Property A, a geometric property guaranteeing coarse embeddability into Hilbert space [Y2]. We shall show that a metric space with (weak) finite decomposition complexity has Property A. As a consequence, any sequence of expanding graphs (as a metric space) does not have (weak) finite decomposition complexity since it does not admit a coarse embedding into Hilbert space.

To prove the main result of this section, it is convenient to work with a characterization of Property A introduced by Dadarlat and Guentner [DG]. A metric family $\mathcal{U}=\{U\}$ is a cover of a metric space $X$ if every $U \in U$ is a metric subspace of $X$ and

$$
X=\bigcup_{U \in U} U
$$

A partition of unity on $X$ subordinate to a cover $U$ is a family of maps $\phi_{U}: X \rightarrow[0,1]$, one for each $U \in \mathcal{U}$, such that each $\phi_{U}$ is supported in $U$ and such that for every $x \in X$,

$$
\sum_{U \in U} \phi_{U}(x)=1 .
$$

We do not require that the sum is finite for any particular $x \in X$.

4.0.8 Definition. A metric family $\mathcal{X}$ is exact if for every $R>0$ and $\varepsilon>0$ and for every $X \in \mathcal{X}$ there is a partition of unity $\left\{\psi_{U}^{X}\right\}$ on $X$ subordinate to a cover $U_{X}$ of $X$ such that the collection

$$
U=\left\{U: U \in U_{X}, \text { some } X\right\}
$$

is a bounded metric family and such that for every $X \in \mathcal{X}$ and every $x, y \in X$,

$$
d(x, y) \leq R \Longrightarrow \sum_{U \in U_{X}}\left|\psi_{U}^{X}(x)-\psi_{U}^{X}(y)\right| \leq \varepsilon .
$$

4.0.9 Remark. Our definition of exactness is equivalent to the notion of an equiexact family of metric spaces introduced by Dadarlat and Guentner (compare [DG], Definitions 2.7 and 2.8). However, we have indexed our partition of unity and cover differently so our definition is not identical to the one in [DG].

For the statements of the next two results, recall that a metric space has bounded geometry if for every $r>0$ there exists an $N=N(r)$ such that every ball of radius $r$ contains at most $N$ points.

4.2 Theorem ([DG], Proposition 2.10). A metric space having Property A is exact. A bounded geometry exact metric space has Property A.

4.3 Theorem. A metric family having (weak) finite decomposition complexity is exact. A bounded geometry metric space having finite decomposition complexity has Property A. 
Proof. We shall present the proof only for FDC, leaving the case of weak FDC to the reader. Let $\mathfrak{E}$ be the collection of exact metric families. It suffices to show that $\mathfrak{E}$ contains the bounded families and is closed under decomposability.

Clearly, $\mathfrak{F}$ contains the bounded families - for $X$ selected from a bounded family the partition of unity comprised of the constant function at 1 , subordinate to the cover $\{X\}$, fulfills the definition.

It remains to check that $\mathfrak{E}$ is closed under decomposability. Let $\mathcal{X}$ be a family and assume $\mathcal{X}$ is decomposable over $\mathfrak{E}$ - for every $r$ there exists $\mathcal{Y} \in \mathfrak{G}$ such that $\mathcal{X}$ is $r$-decomposable over $\mathcal{Y}$. We shall apply [DG], Theorem 4.4, to show that $\mathcal{X} \in \mathfrak{G}$. Let $\delta>0$. Select $r$ large enough so that $r \delta \geq 2$ and obtain $y$ as above. Translating the notion of decomposability into the language of [DG] we see that $y$ is an equi-exact family with the property that every $X \in \mathcal{X}$ admits an $r$-separated cover, the pieces of which belong to $\mathcal{Y}$. Thus, the hypotheses of [DG], Theorem 4.4, are satisfied and we conclude that $\mathcal{X}$ is an equi-exact family. In other words, $\mathcal{X} \in \mathfrak{F}$.

4.0.10 Remark. Theorem 4.4 of $[D G]$ is stated for a single metric space. The same argument can be used to verify that it applies to a metric family.

\section{Further examples}

Additional examples of groups having finite decomposition complexity are readily exhibited based on our results. In this section, we prove that all countable elementary amenable groups, all countable subgroups of almost connected Lie groups, and all countable subgroups of $\operatorname{GL}(n, R)$ for any commutative ring $R$ with unit have finite decomposition complexity.

5.1. Amenable groups. The class of elementary amenable groups is the smallest class of countable discrete groups containing all finite groups and all (countable) abelian groups, and closed under the formation of subgroups, quotients, extensions and direct unions.

5.1.1 Proposition ([C]). The class of elementary amenable groups is the smallest class of countable discrete groups containing all finite groups and all (countable) abelian groups and closed under the formation of extensions and direct unions.

Sketch of proof. Define a class of groups $\mathfrak{Q}$ by transfinite recursion as follows: $\mathfrak{A}_{0}$ is the class of all finite and countable abelian groups; for a successor ordinal $\alpha$ define $\mathfrak{U}_{\alpha}$ to be the class of all groups obtained as a (countable) direct union or extension of groups in $\mathfrak{U}_{\alpha-1}$; for a limit ordinal $\alpha$ define $\mathfrak{A}_{\alpha}=\bigcup_{\beta<\alpha} \mathfrak{A}_{\beta}$; finally, $\mathfrak{A}$ is the collection of groups belonging to some $\mathfrak{A}_{\alpha}$.

From its construction $\mathfrak{U}$ is closed under extensions and (countable) direct unions, and is clearly contained in the collection of elementary amenable groups. It remains 
to show that $\mathfrak{U}$ is closed under subgroups and quotients. Indeed, it is readily verified by transfinite induction that each $\mathfrak{U}_{\alpha}$ is closed under these operations.

5.1.2 Theorem. Elementary amenable groups have finite decomposition complexity.

Proof. We have observed that the class of countable discrete groups having finite decomposition complexity is closed under the formation of extensions and direct unions. Finite groups have finite decomposition complexity, as do (countable) abelian groups. Indeed, a (countable) abelian group is the direct union of its finitely generated subgroups which, according to their general structure theory, have finite decomposition complexity.

5.1.3 Question. Does every countable amenable group have FDC? In particular, does a Grigorchuk group of intermediate growth have FDC?

5.2. Nearly linear groups. A linear group is a group isomorphic to a subgroup of $\mathrm{GL}(n, K)$ for some field $K$. In the companion paper to this note, we proved that a countable linear group has FDC [GTY], Theorem 3.0.1. In this section our first goal is to give two natural generalizations of this result to groups which are 'nearly' linear.

5.2.1 Theorem. A countable subgroup of an almost connected Lie group has finite decomposition complexity.

Proof. We have seen that linear groups have FDC, that FDC is stable under extensions and that (countable) abelian groups have FDC. Thus, the proof is the same as the proof of [GHW], Theorem 6.5: a group as in the statement is realized as an extension with finite quotient and with kernel a subgroup of a connected Lie group; a subgroup of a connected Lie group is realized as an extension with linear quotient and abelian kernel.

5.2.2 Theorem. Let $R$ be a commutative ring with unit. A countable subgroup of $\mathrm{GL}(n, R)$ has finite decomposition complexity.

The proof is based on the following piece of commutative algebra.

5.2.3 Lemma. Let $R$ be a finitely generated commutative ring with unit and let $\mathfrak{n}$ be the nilpotent radical of $R$,

$$
\mathfrak{n}=\left\{r \in R: r^{n}=0 \text { for some } n \in \mathbb{N}\right\} .
$$

The quotient ring $S=R / \mathfrak{n}$ contains a finite number of prime ideals $\mathfrak{p}_{1}, \ldots, \mathfrak{p}_{n}$ such that the diagonal map

$$
S \rightarrow S / \mathfrak{p}_{1} \oplus \cdots \oplus S / \mathfrak{p}_{n}
$$

embeds $S$ into a finite direct sum of domains. 
Proof. This classical fact is a consequence of the Associated Prime Theorem which states that the set of associated primes of a finitely generated module over a Noetherian ring is finite [Ei], Theorem 3.1. Here, the module is the ring itself which is Noetherian since it is finitely generated. The mentioned theorem then says that $R$ has finitely many minimal prime ideals $\mathfrak{p}_{1}, \ldots, \mathfrak{p}_{n}$. The conclusion follows from the fact that their intersection is $\mathfrak{\pi}$.

Proof of Theorem 5.2.2. In views of Proposition 3.2.1, it is enough to treat the case of $\mathrm{GL}_{n}(R)$, where $R$ is finitely generated. With $\mathfrak{n}$ and $S$ as in the previous lemma, we have an exact sequence

$$
1 \rightarrow I+M_{n}(\mathfrak{n}) \rightarrow \mathrm{GL}(n, R) \rightarrow \mathrm{GL}(n, S) \rightarrow 1,
$$

in which $I+M_{n}(\mathfrak{n})$ is nilpotent, and therefore has finite decomposition complexity by Corollary 3.2.5. In the notation of the previous lemma, we have

$$
\operatorname{GL}(n, S) \rightarrow \operatorname{GL}\left(n, S / \mathfrak{p}_{1}\right) \times \cdots \times \operatorname{GL}\left(n, S / \mathfrak{p}_{n}\right) .
$$

So, the quotient has finite decomposition complexity by [GTY], Theorem 3.0.1.

Our next goal in this section is to provide a simplified proof, based on essentially the same ideas, of the following result due to Matsnev [Ma]; it was an important piece of the proof of FDC for linear groups presented in [GTY]. Before turning to the statement, we recall the basic setup. A discrete norm on a field $K$ is a map $\gamma: K \rightarrow[0, \infty)$ satisfying, for all $x, y \in K$,

(1) $\gamma(x)=0 \Longleftrightarrow x=0$,

(2) $\gamma(x y)=\gamma(x) \gamma(y)$,

(3) $\gamma(x+y) \leq \max \{\gamma(x), \gamma(y)\}$,

and for which the range of $\gamma$ on $K^{\times}$is a discrete subgroup of the multiplicative group $(0, \infty)$. Given a discrete norm we define, following [GHW], a (pseudo)-length function $\ell_{\gamma}$ on $\operatorname{GL}(n, K)$ as follows:

$$
\ell_{\gamma}(g)=\log \max _{i j}\left\{\gamma\left(g_{i j}\right), \gamma\left(g^{i j}\right)\right\},
$$

where $g_{i j}$ and $g^{i j}$ are the matrix coefficients of $g$ and $g^{-1}$, respectively.

5.2.4 Proposition (Matsnev). Let $\gamma$ be a discrete norm on a field $K$. The group $\mathrm{GL}(n, K)$, equipped with the (left-invariant pseudo-)metric induced by $\ell_{\gamma}$, is in $\mathfrak{D}_{\mathrm{fin}}$.

Let $\gamma$ be a discrete norm on a field $K$. For the proof of the proposition we shall introduce some subgroups of GL $(n, K)$. The subset

$$
\mathcal{O}=\{x \in K: \gamma(x) \leq 1\}
$$


is a subring of $K$, the ring of integers; the subset

$$
\mathfrak{m}=\{x \in K: \gamma(x)<1\}
$$

is a principal ideal in $\mathcal{O}$; a generator $\pi$ of $\mathfrak{m}$ is a uniformizer. Fix a uniformizer $\pi$. Let $D$ denote the subgroup of diagonal matrices with powers of the uniformizer on the diagonal and let $U$ denote the unipotent upper triangular matrices. Observe that $D$ normalizes $U$ so that $T=D U$ is also a subgroup (namely the group upper triangular matrices). Restrict the length function $\ell_{\gamma}$ to each subgroup and equip each with the associated (left-invariant pseudo-)metric (which is in fact the subspace pseudo-metric from $G)$.

5.2.5 Lemma. The group $U$ has asymptotic dimension zero. In particular, $U \in \mathfrak{D}_{1}$.

Proof. The dilation by (a nonzero) $\theta \in K$ is the function $\Theta: U \rightarrow U$ defined by

$$
\Theta(u)_{i j}=\theta^{j-i} u_{i j}
$$

the entries on the $k^{\text {th }}$-superdiagonal of $n$ are multiplied by $\theta^{k}$. (For $k=0, \ldots, n-1$ the $k^{\text {th }}$-superdiagonal of an $n \times n$ matrix consists of the positions $(i, j)$ for which $j-i=k$.) The formula for matrix multiplication shows that $\Theta$ is an endomorphism of $U$. Further, it is an automorphism with inverse the dilation by $\theta^{-1}$.

Fix $\theta \in K$ of norm greater than one - the inverse of a uniformizer will do. Let $U_{0}$ be the subgroup of $U$ comprised of elements of length zero, and define a sequence of subgroups of $U$ by $U_{k}=\Theta\left(U_{k-1}\right)$. We shall show that

$$
B(1, k \log \gamma(\theta)) \subset U_{k} \subset B(1, k(n-1) \log \gamma(\theta)) .
$$

The lemma follows immediately. Indeed, $U$ is the union of the cosets of $U_{k}$ and the family of these cosets is both bounded and $r$-disjoint, provided $k \log \gamma(\theta)>r$.

In order to verify (5.2) observe that the length function on $U$ is given by

$$
\ell_{\gamma}(u)=\log \max _{i<j}\left\{1, \gamma\left(u_{i j}\right), \gamma\left(u^{i j}\right)\right\} .
$$

For the first inclusion in (5.2) suppose $\ell_{\gamma}(u) \leq k \log \gamma(\theta)$ so that in particular $\gamma\left(u_{i j}\right) \leq \gamma(\theta)^{k}$ for all $i<j$. The non-diagonal $(i, j)$ entry of $\Theta^{-k}(u)$ is $u_{i j} \theta^{k(i-j)}$ so that each has norm at most one. Elementary properties of the norm and (5.3) show that this implies $\Theta^{-k}(u) \in U_{0}$, or $u \in U_{k}$.

The second inclusion in (5.2) follows by induction from

$$
\ell_{\gamma}(\Theta(u)) \leq \ell_{\gamma}(u)+(n-1) \log \gamma(\theta) .
$$

To verify this inequality, note that the non-diagonal $(i, j)$ entry of $\Theta(u)$ is $u_{i j} \theta^{j-i}$ which has norm bounded by $\gamma\left(u_{i j}\right) \gamma(\theta)^{n-1}$. Since $\Theta$ is an automorphism a similar statement applies to the entries of $\Theta(u)^{-1}=\Theta\left(u^{-1}\right)$. The inequality now follows from (5.3). 
5.2.6 Lemma. The group $T$ is in $\mathfrak{D}_{n+1}$.

Proof. Observe that $D \cong \mathbb{Z}^{n}$, and that the restriction of $\ell_{\gamma}$ to $D$ is a proper length function - indeed it corresponds (up to a multiplicative factor) with the supremum norm on $\mathbb{Z}^{n}$ :

$$
\ell_{\gamma}(a)=\max \left|k_{i}\right| \cdot \log \gamma\left(\pi^{-1}\right),
$$

where $a$ is the diagonal matrix with entries $\pi^{k_{i}}$. Hence $D$ is in $\mathfrak{D}_{n}$. It remains to check, as an application of fibering, that $T$ is indeed in $\mathfrak{D}_{n+1} \cdot{ }^{6}$

We require two observations. First, the map $T \rightarrow D$ associating to each matrix in $T$ the matrix of its diagonal entries is a contraction. Indeed, it is a homomorphism and from the definition of $\ell_{\gamma}$ we see that it decreases length. Second, if $B \subset D$ is a bounded subset and $b_{1} \in B$ then the subset $b_{1} U \subset B U$ is diam( $\left.B\right)$-coarsely dense. Indeed, if $b u \in B U$ then $d\left(b u, b u b^{-1} b_{1}\right) \leq \operatorname{diam}(B)$ and, since $D$ normalizes $U$,

$$
b u b^{-1} b_{1}=b_{1}\left(b_{1}^{-1} b\right) u\left(b^{-1} b_{1}\right) \in b_{1} U .
$$

We conclude by applying Theorem 3.1.4 or, more accurately, the subsequent Remark 3.1 .5 to the map $T \rightarrow D$.

Proof of Proposition 5.2.4. The inclusion of $T$ in $G$ is isometric. Further, it is metrically onto in the sense that every element of $G$ is at distance zero from an element of $T$. Indeed, let $H$ be the subgroup of those $g \in \operatorname{GL}(n, K)$ for which the entries of $g$ and $g^{-1}$ are in $\mathcal{O}$. Then $G=T H$ [GHW], Lemma 4.5, and elementary calculations show that every $h \in H$ has length zero. Hence, if $g=t h$ then $d(t, g)=\ell(h)=0$. Observe that we do not assume $K$ is locally compact.

Finally, for the convenience of the reader we shall deduce the following consequence of Proposition 5.2.4; while a special case of [GTY], Theorem 3.0.1, the proof involves similar ideas and serves to illustrate the more general result.

5.2.7 Proposition. The group $\operatorname{GL}\left(n, \mathbb{Z}\left[X_{1}, \ldots, X_{m}\right]\right)$ belongs to $\mathfrak{D}_{\omega+\text { fin. For a prime }}$ power $q$ and $\mathbb{F}_{q}$ the field with $q$ elements, the group $\operatorname{GL}\left(n, \mathbb{F}_{q}\left[X_{1}, \ldots, X_{m}\right]\right)$ has finite asymptotic dimension. (Both assertions are true for every $n \geq 1$ and $m \geq 0$.)

We prepare for the proof by describing the relevant norms on rational function fields. Let us agree that henceforth $K$ stands for either $\mathbb{F}_{q}$ or $\mathbb{Q}$. In the case of a single indeterminant we define, for a nonzero $P \in K[X]$,

$$
\gamma(P)=e^{\operatorname{deg}(P)}
$$

together with the convention $\gamma(0)=0$ this determines uniquely a discrete norm on $K(X)$. We view $K[X] \subset K(X)$, and $\operatorname{GL}(n, K[X]) \subset \mathrm{GL}(n, K(X))$; we equip the latter group with the length function associated by (5.1) to the norm (5.4).

\footnotetext{
${ }^{6}$ Since $D \subset T$ isometrically, if $T$ is in $\mathfrak{D}_{\alpha}$ then necessarily $\alpha \geq n$. An argument more refined than the one we present here achieves this bound: indeed $T \in \mathfrak{D}_{n}$.
} 
We generalize to the case of several indeterminants by defining, in analogy with (5.4), norms on $K\left(X_{1}, \ldots, X_{m}\right)$ reflecting the degree in each of the various indeterminants. ${ }^{7}$ We then view

$$
\begin{aligned}
\mathrm{GL}\left(n, K\left[X_{1}, \ldots, X_{m}\right]\right) & \\
\hookrightarrow & \mathrm{GL}\left(n, K\left(X_{1}, \ldots, X_{m}\right)\right) \times \cdots \times \mathrm{GL}\left(n, K\left(X_{1}, \ldots, X_{m}\right)\right) ;
\end{aligned}
$$

here the factors are equipped with the length functions associated to the various norms according to (5.1), and the length function on the product is the sum.

In the following proof we shall denote by $\ell^{\prime}$ the length function $\mathrm{GL}(n, K[X])$ inherits from $\operatorname{GL}(n, K(X))$; in the multi-variable case, we shall denote by $\ell^{\prime}$ the length function $\operatorname{GL}\left(n, K\left[X_{1}, \ldots, X_{m}\right]\right)$ inherits from the inclusion (5.5). This is done for compatibility of notation with the relevant results from [GTY].

Proof. We begin with the case of a finite field. In the case of a single indeterminant it suffices, by the previous discussion and Proposition 5.2.4, to show that the length function (5.1) on $\operatorname{GL}\left(n, \mathbb{F}_{q}(X)\right)$ restricts to a proper length function on $\operatorname{GL}\left(n, \mathbb{F}_{q}[X]\right)$. But, this is clear: in view of (5.1) and (5.4) bounding $\ell^{\prime}(g)$ bounds the degree of the polynomial entries of $g$ and, since the field $\mathbb{F}_{q}$ is finite, there are only finitely many polynomials of a given degree. In the case of several indeterminants the length function on the product appearing in (5.5) restricts to a proper length function on $\operatorname{GL}\left(n, \mathbb{F}_{q}\left[X_{1}, \ldots, X_{m}\right]\right)$ for the same reason.

The case of $\mathbb{Z}$ is more involved - because there are infinitely many polynomials in $\mathbb{Z}[X]$ of a given degree the length function $\ell^{\prime}$ will not be proper. We shall consider only the case of a single indeterminant and shall rely on the permanence result [GTY], Lemma 3.3.1. In the notation of that lemma, let $r=e^{k}$. Evaluation at a transcendental provides a field embedding $\mathbb{Q}(X) \hookrightarrow \mathbb{C}$ and an embedding $\mathrm{GL}(n, \mathbb{Q}(X)) \hookrightarrow \mathrm{GL}(n, \mathbb{C})$. Fixing distinct transcendental elements $t_{0}, \ldots, t_{k} \in \mathbb{C}$ we obtain a diagonal embedding

$$
\mathrm{GL}(n, \mathbb{Z}[X]) \hookrightarrow \mathrm{GL}(n, \mathbb{Q}(X)) \hookrightarrow \mathrm{GL}(n, \mathbb{C}) \times \cdots \times \mathrm{GL}(n, \mathbb{C}) .
$$

Equip each factor on the right with the length function coming from the operator norm as in [GHW], [GTY]; the length function on the product is the sum; denote by $\ell_{r}$ the length function $\operatorname{GL}(n, \mathbb{Z}[X])$ inherits from the inclusion. Now, with its length function $\operatorname{GL}(n, \mathbb{C})$ belongs to $\mathfrak{D}_{\text {fin }}$, so that $\operatorname{GL}(n, \mathbb{Z}[X])$ with the length function $\ell_{r}$ does as well. By [GTY], Lemma 3.3.1, we are reduced to showing that the collection of $g \in \mathrm{GL}(n, \mathbb{Z}[X])$ satisfying the inequalities

$$
\ell^{\prime}(g) \leq r=e^{k}, \quad \ell_{r}(g) \leq s
$$

\footnotetext{
${ }^{7}$ These are in fact the norms of type (1) appearing in the proof of [GTY], Lemma 3.1.5. Precisely, in the case of two indeterminants, if we identify $K(X, Y)$ and $K(X)(Y)$ then the extension to $K(X, Y)$ of the norm on $K(X)$ determined by (5.4) is itself determined by the formula $\gamma(P)=\max \left\{\gamma\left(P_{j}\right)\right\}$ where $P(X, Y)=P_{0}(X)+P_{1}(X) Y+\cdots+P_{n}(X) Y^{n}$ and the $P_{j} \in K(X)$. In particular, if $P \in K[X, Y]$ so that the $P_{j} \in K[X]$ then $\gamma(P)$ is exactly the degree of $P$ 'with respect to $X^{\prime}$ '. See also [GHW], Theorem 2.2.
} 
is finite, for every $s$. Arguing exactly as in the first part of this proof, we conclude from the first inequality that the polynomial entries of such $g$ have degree bounded by $k$. It follows from the second inequality that if $P \in \mathbb{Z}[X]$ is a polynomial entry of such $g$ then for every transcendental $t_{i}$ the absolute value of $P\left(t_{i}\right)$ is bounded by $s$. In other words, we have reduced to showing that the set

$$
\left\{P \in \mathbb{Z}[X]_{k}:\left|P\left(t_{i}\right)\right| \leq s \text { for } i=0, \ldots, k\right\}
$$

is finite, where $\mathbb{Z}[X]_{k}$ is the set of polynomials of degree at most $k$. This is, however, straightforward: the assignment

$$
P \mapsto\left(P\left(t_{0}\right), \ldots P\left(t_{k}\right)\right)
$$

defines an isomorphism of complex vector spaces $\mathbb{C}[X]_{k} \rightarrow \mathbb{C}^{k+1}$ - identifying a polynomial $P \in \mathbb{C}[X]_{k}$ with the column vector formed by its coefficients it is given by the Vandermonde matrix corresponding to the distinct transcendentals $t_{0}, \ldots, t_{k}$ - and $\mathbb{Z}[X]_{k} \subset \mathbb{C}[X]_{k}$ is discrete.

\section{References}

[A] J. M. Alonso, T. Brady, D. Cooper, V. Ferlini, M. Lustig, M. Mihalik, M. Shapiro, and H. Short, Notes on word hyperbolic groups. In Group theory from a geometrical viewpoint, World Scientific, Singapore 1991, 3-63. Zbl 0849.20023 MR 1170363

[AD] G. N. Arzhantseva and T. Delzant, Examples of random groups. Preprint 2008, 2011. http://www.mat.univie.ac.at/\%7Earjantseva/Abs/random.pdf

[BD1] G. Bell and A. Dranishnikov, On asymptotic dimension of groups. Algebr. Geom. Topol. 1 (2001), 57-71. Zbl 1008.20039 MR 1808331

[BD2] G. Bell and A. Dranishnikov, On asymptotic dimension of groups acting on trees. Geom. Dedicata 103 (2004), 89-101. Zbl 1131.20032 MR 2034954

[C] C. Chou, Elementary amenable groups. Illinois J. Math. 24 (1980), 396-407. Zbl 0439.20017 MR 573475

[DG] M. Dadarlat and E. Guentner, Constructions preserving Hilbert space uniform embeddability of discrete groups. Trans. Amer. Math. Soc. 355 (2003), 3253-3275. Zbl 1028.46104 MR 1974686

[DS] A. Dranishnikov and J. Smith, Asymptotic dimension of discrete groups. Fund. Math. 189 (2006), 27-34. Zbl 1100.20034 MR 2213160

[DZ] A. Dranishnikov and M. Zarichnyi, Universal spaces for asymptotic dimension. Topology Appl. 140 (2004), 203-225. Zbl 1063.54027 MR 2074917

[Ei] D. Eisenbud, Commutative algebra. Graduate Texts in Math. 150, Springer-Verlag, New York 1995. Zbl 0819.13001 MR 1322960

[G1] M. Gromov, Geometric group theory. Vol. 2: Asymptotic invariants of infinite groups. London Math. Soc. Lecture Note Ser. 182, Cambridge University Press, Cambridge 1993. Zbl 0841.20039 MR 1253544 
[G2] M. Gromov, Spaces and questions. In Visions in Mathematics: GAFA 2000 Special Volume, Part I, Birkhäuser, Basel 2000, 118-161. Zbl 1006.53035 MR 1826251

[G3] M. Gromov, Random walk in random groups. Geom. Funct. Anal. 13 (2003), 73-146. Zbl 1122.20021 MR 1978492

[G] E. Guentner, Permanence in coarse geometry. Preprint 2010.

[GHW] E. Guentner, N. Higson, and S. Weinberger, The Novikov conjecture for linear groups. Publ. Math. Inst. Hautes Études Sci. 101 (2005), 243-268. Zbl 1073.19003 MR 2217050

[GTY] E. Guentner, R. Tessera, and G. Yu, A notion of geometric complexity and its applications to topological rigidity. Invent. Math. 189 (2012), 315-357. Zbl 1257.57028 MR 2947546

[Ma] D. Matsnev, The Baum-Connes conjecture and proper group actions on affine buildings. Preprint 2007 arXiv:math/0703923

[RY1] A. Ranicki and M. Yamasaki, Controlled $K$-theory. Topology Appl. 61 (1995), 1-59. Zbl 0835.57013 MR 1311017

[RY2] A. Ranicki and M. Yamasaki, Controlled L-theory. In Exotic homology manifolds Oberwolfach 2003, Geom. Topol. Monogr. 9, Geom. Topol. Publ., Coventry 2006, 105-153. Zbl 1127.57014 MR 2222493

[R1] J. Roe, Lectures on coarse geometry. Univ. Lecture Ser. 31, Amer. Math. Soc., Providence, RI, 2003. Zbl 1042.53027 MR 2007488

[Y1] G. Yu, The Novikov conjecture for groups with finite asymptotic dimension. Ann. of Math. (2) 147 (1998), 325-355. Zbl 0911.19001 MR 1626745

[Y2] G. Yu, The coarse Baum-Connes conjecture for spaces which admit a uniform embedding into Hilbert space. Invent. Math. 139 (2000), 201-240. Zbl 0956.19004 MR 1728880

Received September 19, 2011; revised November 30, 2011

E. Guentner, Department of University of Hawai 'i at Mānoa, Department of Mathematics, 2565 McCarthy Mall, Honolulu, HI 96822, U.S.A.

E-mail: erik@math.hawaii.edu

R. Tessera, ENS de Lyon, UMPA, UMR CNRS 5669, 46 allée d'Italie, 69364 Lyon Cedex 07, France

E-mail: tessera@phare.normalesup.org

G. Yu, Department of Mathematics, Mailstop 3368, Texas A\&M University, College Station, TX 77843-3368, U.S.A.

E-mail: guoliangyu@math.tamu.edu 